\title{
The Basolateral Nucleus of the Amygdala Is Necessary to Induce the Opposing Effects of Stressful Experience on Learning in Males and Females
}

\author{
Jaylyn Waddell, Debra A. Bangasser, and Tracey J. Shors \\ Department of Psychology and Center for Collaborative Neuroscience, Rutgers University, Piscataway, New Jersey 08854
}

\begin{abstract}
The basolateral nucleus of the amygdala (BLA) has been implicated in the modulation of learning after stress. Acute inescapable stress enhances classical eyeblink conditioning in male rats, whereas the same stressor impairs eyeblink conditioning in female rats. The experiments here directly assessed whether inactivation of the BLA during stress exposure would block both the stress-induced facilitation in males and the retardation of eyeblink conditioning in females. To this end, the BLA was temporarily inactivated by infusion of the GABA agonist muscimol before acute stressor exposure. All rats were trained in a different context $24 \mathrm{~h}$ later. Males infused with muscimol before the stressful event did not exhibit facilitated eyeblink conditioning, whereas those infused with the vehicle emitted more conditioned responses than unstressed males. Females infused with muscimol before stress did not express a deficit in conditioning, whereas those infused with vehicle and stressed emitted fewer conditioned responses than unstressed vehicle controls. These data demonstrate that neuronal activity within the BLA during stress exposure is necessary to modulate learning $24 \mathrm{~h}$ later in a new context. Thus, the BLA is necessary to induce the long-term effect of stressful experience on conditioning regardless of sex and direction of modulation.
\end{abstract}

Key words: stress; eyeblink conditioning; basolateral amygdala; muscimol; sex differences; pavlovian conditioning

\section{Introduction}

Acute stress facilitates acquisition of many associative learning tasks, such as fear conditioning (Maier, 1990; Cordero et al., 2003; Rodríguez Manzanares et al., 2005) and eyeblink conditioning (Shors et al., 1992; Beylin and Shors, 1998; Neufeld and Mintz, 2001; Shors, 2001). Based on its established role in emotions and emotional learning, the basolateral nucleus of the amygdala (BLA) is a structure likely to modulate learning after stress. The BLA is critically involved in the stress-induced modulation of classical conditioning (Neufeld and Mintz, 2001; Rodríguez Manzanares et al., 2005). Antagonism of NMDA receptors within the BLA at the time of stress exposure blocked the facilitation of eyeblink conditioning (Shors and Mathew, 1998). Similarly, a stress-induced enhancement of contextual fear conditioning was abolished by intra-amygdala administration of the GABA agonist midazolam before stress exposure (Rodríguez Manzanares et al., 2005). In tasks such as passive avoidance, the BLA positively modulates memory consolidation (McGaugh and Roozendaal, 2002; Roozendaal et al., 2002) and also modulates activity in the hippocampus during learning and stress (Vazdar-

\footnotetext{
Received Dec. 20, 2007; accepted April 3, 2008.

This work was supported by National Institutes of Health (NIH)-National Institute of Mental Health (NIMH) Grant MH59970 and National Science Foundation Grant IOB-0444364 (T.J.S.) and NIH-NIMH Grant MH019957 (J.W.).

Correspondence should be addressed to Dr. Tracey J. Shors, Department of Psychology, Rutgers University, 152 Frelinghuysen Road, Piscataway, NJ 08854. E-mail: shors@rutgers.edu.

D. A. Bangasser's present address: Children's Hospital of Philadelphia, Philadelphia, PA 19104

D0I:10.1523/JNEUROSCI.1129-08.2008

Copyright $\odot 2008$ Society for Neuroscience $\quad$ 0270-6474/08/285290-05\$15.00/0
}

janova and McGaugh, 1999; Kim et al., 2001; Huff and Rudy, 2004; Blankenship et al., 2005; Korz and Frey, 2005). In general, an intact amygdala is necessary for stress-induced facilitation of classical fear and eyeblink conditioning (Shors and Mathew, 1998; Neufeld and Mintz, 2001; Rodríguez Manzanares et al., 2005). It is important to note that these reports were conducted exclusively in male animals (Shors and Mathew, 1998; Neufeld and Mintz, 2001; Rodríguez Manzanares et al., 2005).

In contrast to males, female rats express a profound deficit in classical eyeblink conditioning after a stressful experience, especially when they are stressed in diestrus and trained in proestrus, when estrogen levels are high (Shors et al., 1998; Wood et al., 2001). The presence of glucocorticoids is necessary to express the facilitation of eyeblink conditioning in males (Beylin and Shors, 2003) but is not necessary for the retarded acquisition expressed by females (Wood et al., 2001). These sex differences may ultimately relate to the circuitry used to modulate learning after stress. Numerous studies find that glucocorticoids injected directly into the BLA enhance aversive learning and memory (Roozendaal et al., 2002). Because the BLA is generally implicated in the facilitation of learning, we asked whether the nucleus might also be necessary for decremented responses after stress. Here we used reversible inactivation of the BLA at the time of the stressor to assess its role in the modulation of associative learning in both male and female rats.

\section{Materials and Methods}

Subjects. Male and cycling female Sprague Dawley rats between 90 and $120 \mathrm{~d}$ of age (obtained from a breeding facility at Rutgers University) 
were used. Rats were housed in groups of three until surgery. After surgery, rats were housed alone in standard plastic "shoebox" cages $(44.5 \mathrm{~cm}$ long, $21.59 \mathrm{~cm}$ wide, and $23.32 \mathrm{~cm}$ high). Rats had ad libitum access to rat chow and water and were maintained on a $12 \mathrm{~h}$ light/dark cycle. All experiments were conducted with full compliance to the rules and regulations specified by the Public Health Service Policy on Humane Care and Use of Laboratory Animals and the National Institutes of Health Guide for the Care and Use of Laboratory Animals.

Surgery. Rats were anesthetized with sodium pentobarbital $(50 \mathrm{mg} / \mathrm{kg}$ for males and $40 \mathrm{mg} / \mathrm{kg}$ for females). After being placed in the stereotaxic instrument, the scalp was cleaned with Betadine, and an incision was made. Guide cannulas (23 gauge; Plastics One, Roanoke, VA) were implanted bilaterally aimed at the basolateral nucleus of the amygdala (anteroposterior, -3.0 ; mediolateral, \pm 4.8 ; dorsoventral, -7.6 from surface of skull). Cannulas and headstages were fixed with dental cement and four jeweler screws. In preparation for eyeblink conditioning, four eyelid electrodes (insulated stainless-steel wire, 0.005 inch) were implanted through the upper eyelid (orbicularis oculi muscle). Rats were allowed a minimum of 1 week recovery before eyeblink conditioning began.

Vaginal cytology. Stages of estrus were monitored daily beginning the day after surgery and a minimum of 5-7 d before training. Sterile cotton swabs were dipped in physiological saline and gently inserted into the vaginal canal, to collect loose epithelial cells. These cells were applied to slides, and stained with a $1 \%$ Toluidine Blue solution. Cells were rinsed and dehydrated with $95 \% \mathrm{EtOH}$. Each phase of the estrous cycle was identified using a microscope. Females were stressed in diestrus and trained in proestrus, when estrogen levels are increasing (Shors et al., 1998; Wood et al., 2001). Animals that failed to exhibit a normal estrous cycle were eliminated from the study.

Infusions. During infusions, stylets were replaced with infusion cannulas protruding $1 \mathrm{~mm}$ past the guide cannula. Infusion cannulas were attached to a microinfusion pump via polyethylene tubes attached to 10 $\mu l$ Hamilton syringes. The syringe and tubes were filled with water, and a small air bubble separated the water from the artificial CSF (ACSF) or muscimol solution. Rats were infused with $0.25 \mu \mathrm{g}$ of muscimol into each hemisphere. The drug was infused at a rate of $0.125 \mu 1 / \mathrm{min}$ over 2 min for a total of $0.25 \mu \mathrm{l}$.

Conditioning chamber acclimation followed by stressor exposure in a novel context. Rats were placed in the conditioning boxes for an acclimation period. During this time, spontaneous eyeblinks were recorded. Rats were then transported to a separate room and infused with either ACSF or muscimol. After infusions, rats were placed in a different context that consisted of a white wooden soundproof box with no illumination. Rats were placed in restraint tubes, and electrodes were attached to the tail. Thirty $1 \mathrm{~s}, 1 \mathrm{~mA}$ stimulations were delivered at $1 \mathrm{~min}$ intervals for $30 \mathrm{~min}$, for a total of 30 tail shocks. Rats in the no-stress condition were infused with ACSF and returned to the home cage.

Trace eyeblink conditioning. Twenty-four hours after stressor exposure, rats were placed in the eyeblink conditioning chambers and trained with the trace eyeblink conditioning procedure for 150 trials/d for 4 consecutive days (600 trials total). The conditioned stimulus (CS) was an $83 \mathrm{~dB}, 250 \mathrm{~ms}$ white noise. The unconditioned stimulus (US) was a 100 ms periorbital shock $(0.65 \mathrm{~mA})$. The CS and US were separated by a 500 $\mathrm{ms}$ trace interval, in which no stimuli were delivered. Trials were presented in blocks of 10 in the following order: one CS-alone trial, four paired trials, one US-alone trial, and four paired trials, with an intertrial interval of $25 \pm 5 \mathrm{~s}$. Conditioned responses (CRs) were eyeblinks that occurred during the $500 \mathrm{~ms}$ trace interval on paired trials and $750 \mathrm{~ms}$ after the CS offset on CS-alone trials. The percentage of CRs emitted across blocks of trials was analyzed using repeated-measures ANOVAs.

\section{Results}

\section{Histology}

After training, rats were deeply anesthetized and transcardially perfused with saline followed by $10 \%$ formalin. Brains were extracted and then postfixed in 10\% formalin for 3-4 $\mathrm{d}$ and then transferred to storage in $30 \%$ sucrose in formalin. Coronal sec- tions $(40 \mu \mathrm{m})$ were taken throughout the BLA using a cryostat. Cannula placements within the BLA were between -2.30 and $-3.30 \mathrm{~mm}$ relative to bregma. Reconstruction of cannula placements for those rats retained in analysis is depicted in Figure 1. Cannulas were judged to be within the BLA if the tips were bilaterally on the dorsal boundary of the lateral nucleus of the amygdala. Site of drug infusion was assessed by track markings of the infusion cannula, which protruded $1 \mathrm{~mm}$ beyond the guide cannula. Rats were excluded from analysis if cannula placements were not within the BLA or if the BLA was damaged by the cannula or the infusion. Each condition was comprised of the following: males, ACSF-no stress $(n=8)$; ACSF-stress $(n=6)$; muscimol (mus)-stress $(n=6)$; females, ACSF-no stress $(n=8)$; ACSF-stress $(n=6)$; mus-stress $(n=8)$.

\section{Behavioral results}

Behavioral results are presented in Figure 2. In Figure 2, $A$ and $C$, eyeblink conditioning trials are presented in 10-trial blocks for the first 50 trials to depict any possible differences between groups in early trials. Thereafter, conditioning trials are presented in 50-trial blocks. ANOVA revealed no significant group differences in spontaneous blinks emitted during the acclimation session in males $\left(F_{(2,17)}<1\right)$. Repeated-measures ANOVA on the first 5 blocks of 10 trials failed to find a significant effect of trial $\left(F_{(4,68)}=1.22 ; p>0.05\right)$ or a trial $\times$ condition interaction $\left(F_{(8,68)}\right.$ $<1)$. The main effect of condition did not reach significance $\left(F_{(2,17)}=3.42 ; p=0.057\right)$. Bonferroni's post hoc tests found no significant differences between groups $(p>0.05)$. A repeatedmeasures ANOVA across the 16 blocks of eyeblink conditioning revealed a significant effect of trial $\left(F_{(15,255)}=19.41 ; p=0.0001\right)$, as males expressed an increase in conditioned responding across trial blocks. The trial $\times$ condition interaction did not reach significance $\left(F_{(30,255)}<1\right)$. The main effect of condition was significant $\left(F_{(2,17)}=5.69 ; p=0.013\right)$. Bonferroni's post hoc tests confirmed that males treated with ACSF before stress exposure expressed more conditioned responses than unstressed rats $(p=$ 0.019 ) or rats treated with muscimol before stress exposure ( $p=$ $0.038)$. Rats treated with muscimol before stress exposure did not differ from unstressed, ACSF-treated controls $(p>0.05)$. Thus, rats treated with muscimol before stress exposure did not express facilitated learning relative to ACSF-treated rats exposed to stress, and performed similarly to unstressed rats.

Analysis of spontaneous blink rates of females during the acclimation session and before conditioning failed to find any group differences $\left(F_{(2,19)}<1\right)$. Analysis of the first 5 blocks of 10 trials found no effect of trial $\left(F_{(4,76)}<1\right)$, nor a trial $\times$ condition interaction $\left(F_{(8,76)}=1.19 ; p>0.05\right)$. The main effect of condition was not significant $\left(F_{(2,19)}=2.76 ; p>0.05\right)$. Thus, stress or drug treatment did not influence early responding. A repeatedmeasures ANOVA across the 16 blocks of eyeblink conditioning depicted in Figure $2 C$ revealed a significant effect of trial $\left(F_{(15,285}\right.$ $=12.13 ; p<0.0001$ ), as females expressed an increase in conditioned responding as training progressed. The trial $\times$ condition interaction did not reach significance $\left(F_{(30,285)}=1.35 ; p>0.05\right)$. The main effect of condition was significant $\left(F_{(2,19)}=5.41 ; p=\right.$ 0.014 ). Bonferroni's post hoc analyses confirmed that female rats treated with ACSF before stress exposure did not express as many conditioned responses as unstressed females $(p=0.042)$ or females treated with muscimol before stress exposure $(p=0.019)$. Rats treated with muscimol before stress exposure did not differ from unstressed female rats $(p>0.05)$. Thus, muscimol before stress exposure abolished the deleterious effect of stress on subsequent eyeblink conditioning. 

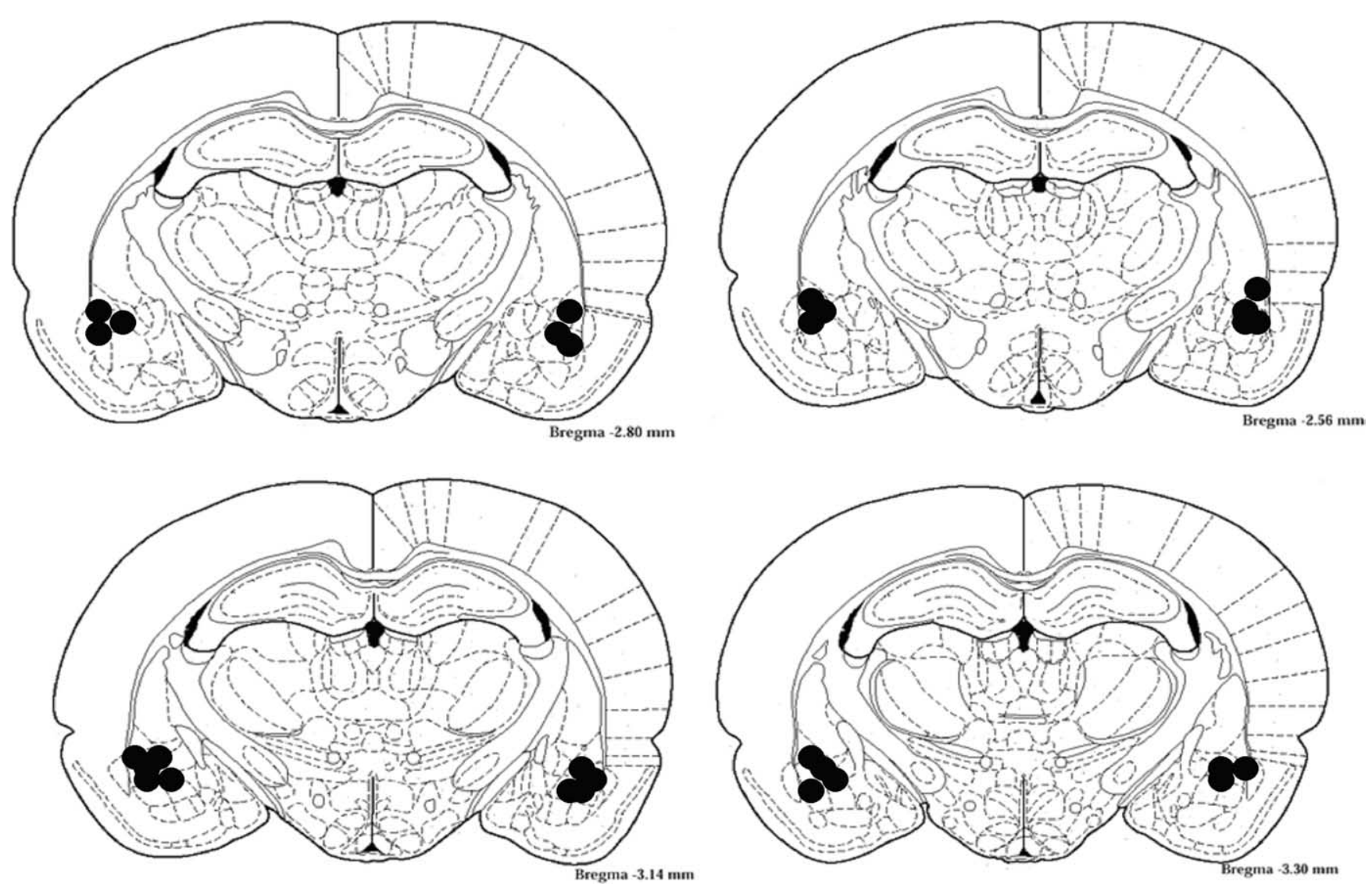

Figure 1. Histology: location of cannula tips for all rats included in statistical analysis. Rats included in analysis were confirmed to have cannulas within -2.30 to $-3.30 \mathrm{~mm}$ relative to bregma. Because of extensive overlap cannula tip locations, not all individual injector locations are indicated. Coronal brain section images were adapted from Paxinos and Watson (1998).

Figure 2, $B$ and $D$, depicts the percentage of rats to reach $60 \%$ conditioned responding on any block of 50 trials. The majority of unstressed females attained this level of conditioned responding $(87.5 \%)$, whereas stress reduced the number of females reaching this criterion (33.3\%). Importantly, a higher percentage of female rats treated with muscimol in the BLA (75\%) during the stressor reached criterion relative to stressed females. Male rats exhibited the opposite pattern of results. Only $50 \%$ of unstressed males reached a criterion of $60 \%$ conditioned responding, whereas all male rats exposed to stress reached criterion. When the BLA was inactive during the stressor, only one-half of the males reached criterion, similar to unstressed males. These results indicate that exposure to the acute stressor is affecting, not only acquisition, but also how many animals attain a learning criterion of $60 \%$ conditioned responses.

\section{Discussion}

In both males and females, BLA inactivation with muscimol during the stressor prevented the modification of classical eyeblink conditioning. These data were somewhat anticipated given the established role of the amygdala in aversive conditioning (LeDoux, 2000; Roozendaal et al., 2002). However, involvement of the BLA in decremented learning after stress in females has not been previously demonstrated. Although the BLA is not necessary for eyeblink conditioning, lesions of the BLA profoundly retard acquisition (Neufeld and Mintz, 2001; Lee and Kim, 2004; Lindquist and Brown, 2004), suggesting that the BLA is involved in the modulation of learning during arousing and fearful conditions (Packard and Cahill, 2001; McGaugh, 2004). Inescapable stress elicits fear that generalizes beyond the context in which inescapable stress was conducted, and this fear is evident before US delivery (Maier, 1990). This generalized fear facilitates aversive conditioning in a novel context in males (Maier, 1990). The present results suggest that activity within the BLA elicited during the stressor may potentiate contextual fear expressed during eyeblink conditioning and thereby enhance acquisition in male rats. Similarly, enhancement of GABAergic inhibition within the BLA during acute restraint stress blocks the stress-induced enhancement of contextual fear conditioning in male rats (Rodríguez Manzanares et al., 2005). These relationships have not been demonstrated in females.

If the BLA is mediating the emotional components of stress exposure and eyeblink conditioning, it follows that fear disrupts eyeblink conditioning in females. Whether fear expressed by stressed females during eyeblink conditioning is different or more persistent than that expressed by unstressed females or males is unknown. The influence of stress on eyeblink conditioning is dependent on different hormones, which may modulate the expression of fear differently in males relative to females. The effect of stress on eyeblink conditioning in females is dependent on the presence of estrogen (Wood and Shors, 1998), and acute stress elicits a prolonged elevation in estrogen levels (Shors et al., 1999). Estrogen receptor agonists can disrupt the conditional inhibition of fear in females (Toufexis et al., 2007), and estrogen modulates many types of learning (e.g., Zurkovsky et al., 2007). The presence of estrogen during training in females exposed to acute stress may be enhancing the expression of fear to a degree that disrupts their ability to acquire the CS-US association or emit adaptively timed CRs. 


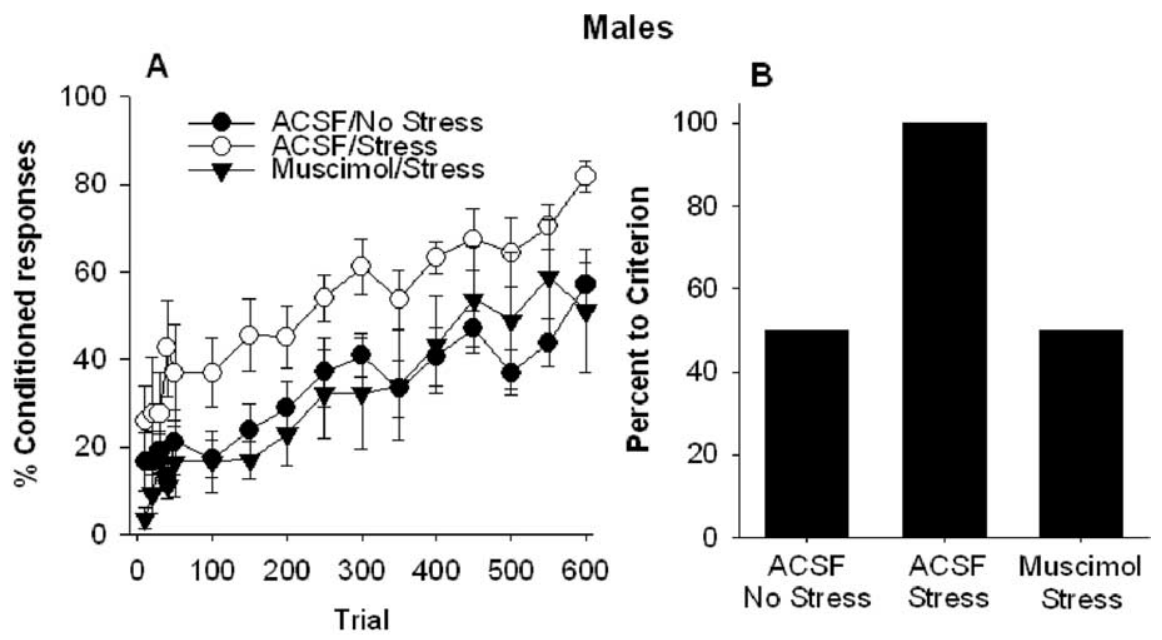

Females
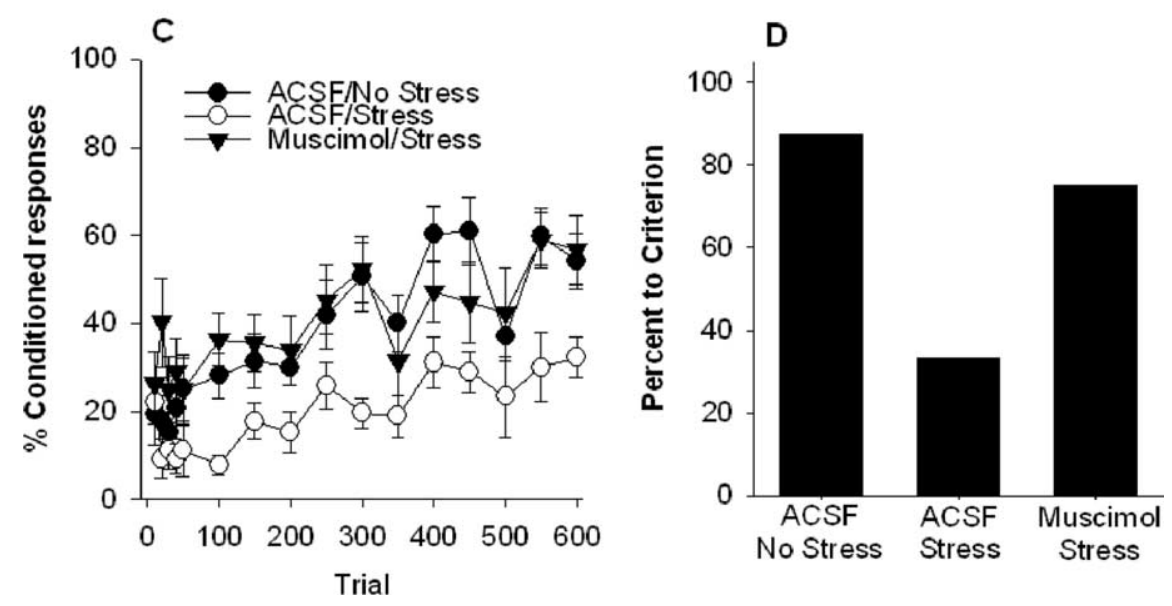

Figure 2. A, Percentage of CRs of male rats in each condition across eyeblink conditioning sessions. Infusion of muscimol into the BLA abolished the stress-induced facilitation of eyeblink conditioning. $\boldsymbol{B}$, Number of male rats reaching a behavioral criterion of $60 \%$ CRs in each drug and stress condition. C, Percentage of CRs of female rats in each condition across eyeblink conditioning sessions. Infusion of muscimol into the BLA abolished the stress-induced disruption of eyeblink conditioning. $\boldsymbol{D}$, Number of female rats reaching a behavioral criterion of $60 \%$ in each drug and stress condition.

Whether the opposing effect of stress on eyeblink conditioning relies on intrinsic amygdala changes is unknown. Exposure to the stressor used here dramatically and persistently impairs multiple-unit activity within the BLA (Shors, 1999), and chronic stress enhances synaptic connectivity in the BLA (Vyas et al., 2006). Furthermore, glucocorticoids within the BLA enhance consolidation of aversive learning (McGaugh and Roozendaal, 2002; Roozendaal et al., 2002) and increase excitability of principal neurons within the BLA (Duvarci and Pare, 2007). To our knowledge, these results are restricted to male rats. Direct examination of sex differences in response to stress within the BLA found that although serotonin and dopamine levels are lower in the amygdala of female than in that of male rats, stress exposure elevates these transmitter levels above those of males (Mitsushima et al., 2006). Females also expressed a larger increase in systemic corticosterone after stress exposure relative to males (Mitsushima et al., 2006), despite the fact that glucocorticoids are not necessary for the effect of stress on eyeblink conditioning in females (Wood et al., 2001). Thus, the female response to stress may be attributable to changes in neural structures sensitive to fluctuation of estrogens, because the deleterious influence of stress on learning occurs when estrogen levels are highest (Wood and Shors, 1998). Whether this possible sensitivity to estrogens is within the BLA or afferent/efferent structures remains unknown.

The hippocampus is also necessary in males and females for the influence of stress on acquisition of classical eyeblink conditioning (Bangasser and Shors, 2007). Thus, the hippocampus is critical for the modulatory role of stress in both sexes beyond its putative role in learning (Bangasser and Shors, 2007). The BLA may interact directly with the hippocampus during the stressor to modify subsequent learning in both males and females. The hippocampus exhibits morphological and excitability changes in response to stress (Galea et al., 1997; Kim et al., 2001; Shors et al., 2001) as well as eyeblink conditioning (Geinisman et al., 2001; Moyer et al., 1996). The BLA modulates neuronal activity within the hippocampus in response to stress exposure (Kim et al., 2001; Korz and Frey, 2005) and many learning tasks, including eyeblink conditioning (Pare, 2003; Huff and Rudy, 2004; Blankenship et al., 2005; McIntyre et al., 2005). The hippocampus is sensitive to fluctuating estrogen levels (Woolley et al., 1990; Yankova et al., 2001); whether estrogen levels directly influence BLA-hippocampal interactions during stress exposure and eyeblink conditioning is unknown. It is possible that inactivation of the BLA at the time of stress exposure may disrupt amygdala-dependent modulation of the hippocampus and, in turn, abolish the influence of stress on learning in both males and females.

The current results indicate that the BLA is critically engaged during a stressful event and can modify learning at least $24 \mathrm{~h}$ later in male and female rats. A direct comparison of neural substrates used by males versus females to respond to stress may provide insight into sex differences in emotional memory, as well as in the prevalence of posttraumatic stress disorder in women (Cahill et al., 2001; Canli et al., 2002; Tolin and Foa, 2006). The results presented here suggest that the amygdala is not only engaged by stress but is necessary to initiate the opposing effect of stressful experience on classical conditioning.

\section{References}

Bangasser DA, Shors TJ (2007) The hippocampus is necessary for enhancements and impairments of learning following stress. Nat Neurosci 10:1401-1403.

Beylin AV, Shors TJ (1998) Stress enhances excitatory trace eyeblink conditioning and opposes acquisition of inhibitory conditioning. Behav Neurosci 112:1327-1338.

Beylin AV, Shors TJ (2003) Glucocorticoids are necessary for enhancing acquisition of associative memories after acute stressful experience. Horm Behav 43:124-131.

Blankenship MR, Huckfeldt R, Steinmetz JJ, Steinmetz JE (2005) The effects of amygdala lesions on hippocampal activity and classical eyeblink conditioning in rats. Brain Res 1035:120-130.

Cahill L, Haier RJ, White NS, Fallon J, Kilpatrick L, Lawrence C, Potkin SG, 
Alkire MT (2001) Sex-related difference in amygdala activity during emotionally influenced memory storage. Neurobiol Learn Mem 75:1-9.

Canli T, Desmond JE, Zhao Z, Gabrieli JD (2002) Sex differences in the neural basis of emotional memories. Proc Natl Acad Sci USA 99:10789-10794.

Cordero MI, Venero C, Kruyt ND, Sandi C (2003) Prior exposure to a single stress session facilitates subsequent contextual fear conditioning in rats. Horm Behav 44:338-345.

Duvarci S, Pare D (2007) Glucocorticoids enhance excitability of principal basolateral amygdala neurons. J Neurosci 27:4482-4491.

Galea LAM, McEwen BS, Tanapat P, Deak T, Spencer RL, Dhabhar FS (1997) Sex differences in dendritic atrophy of CA3 pyramidal neurons in response to chronic restraint stress. Neuroscience 81:689-697.

Geinisman Y, Berry RW, Disterhoft JF, Power JM, Van der Zee EA (2001) Associative learning elicits the formation of multiple-synapse boutons. J Neurosci 21:5568-5573.

Huff NC, Rudy JW (2004) The amygdala modulates hippocampusdependent context memory formation and stores cue-shock associations. Behav Neurosci 118:53-62.

Kim JJ, Lee HJ, Han J-S, Packard MG (2001) Amygdala is critical for stressinduced modulation of hippocampal long-term potentiation and learning. J Neurosci 21:5222-5228.

Korz V, Frey JU (2005) Bidirectional modulation of hippocampal longterm potentiation under stress and no-stress conditions in basolateral amygdala-lesioned and intact rats. J Neurosci 25:7393-7400.

LeDoux JE (2000) Emotion circuits in the brain. Annu Rev Neurosci 23:155-184.

Lee T, Kim JJ (2004) Differential effects of cerebellar, amygdalar, and hippocampal lesions on classical eyeblink conditioning in rats. J Neurosci 24:3242-3250.

Lindquist DH, Brown TH (2004) Amygdalar NMDA receptors control the expression of associative reflex facilitation and three other conditional responses. Behav Neurosci 118:36-52.

Maier SF (1990) The role of fear in mediating the shuttle escape learning deficit produced by inescapable shock. J Exp Psychol Anim Behav Process 16:137-150.

McGaugh JL (2004) The amygdala modulates the consolidation of memories of emotionally arousing experiences. Annu Rev Neurosci 27:1-28.

McGaugh JL, Roozendaal B (2002) Role of adrenal stress hormones in forming lasting memories in the brain. Curr Opin Neurobiol 12:205-210.

McIntyre CK, Miyashita T, Setlow B, Marjon KD, Steward O, Guzowski JF, McGaugh JL (2005) Memory-influencing intra-basolateral amygdala drug infusions modulate expression of Arc protein in the hippocampus. Proc Natl Acad Sci USA 102:10718-10723.

Mitsushima D, Yamada K, Takase K, Funabashi T, Kimura F (2006) Sex differences in the basolateral amygdala: the extracellular levels of serotonin and dopamine, and their responses to restraint stress in rats. Eur J Neurosci 24:3245-3254.

Moyer Jr JR, Thompson LT, Disterhoft JF (1996) Trace eyeblink conditioning increases CA1 excitability in a transient and learning-specific manner. J Neurosci 16:5536-5546.

Neufeld M, Mintz M (2001) Involvement of the amygdala in classical conditioning of eyeblink response in the rat. Brain Res 889:112-117.

Packard MG, Cahill L (2001) Affective modulation of multiple memory systems. Curr Opin Neurobiol 11:752-756.

Pare D (2003) Role of the basolateral amygdala in memory consolidation. Prog Neurobiol 70:409-420.
Paxinos G, Watson C (1998) The rat brain in stereotaxic coordinates, Ed 4. New York: Academic.

Rodríguez Manzanares PA, Isoardi NA, Carrer HF, Molina VA (2005) Previous stress facilitates fear memory, attenuates GABAergic inhibition, and increases synaptic plasticity in the rat basolateral amygdala. J Neurosci 25:8725-8734.

Roozendaal B, Brunson KL, Holloway BL, McGaugh JL, Baram TZ (2002) Involvement of stress-released corticotrophin-releasing hormone in the basolateral amygdala in regulating memory consolidation. Proc Natl Acad Sci USA 99:13908-13913.

Shors TJ (1999) Acute stress and re-exposure to the stressful context suppress spontaneous unit activity in the basolateral amygdala via NMDA receptor activation. NeuroReport 10:2811-2815.

Shors TJ (2001) Acute stress rapidly and persistently enhances memory formation in the male rat. Neurobiol Learn Mem 75:10-29.

Shors TJ, Mathew PR (1998) NMDA receptor antagonism in the lateral/ basolateral but not central nucleus of the amygdala prevents the induction of facilitated learning in response to stress. Learn Mem 5:220-230.

Shors TJ, Weiss C, Thompson RF (1992) Stress-induced facilitation of classical conditioning. Science 257:537-539.

Shors TJ, Lewczyk C, Pacynski M, Mathew PR, Pickett J (1998) Stages of estrous mediate the stress-induced impairment of associative learning in the female rat. Learn Mem 9:419-423.

Shors TJ, Pickett J, Wood G, Paczynski M (1999) Acute stress persistently enhances estrogen levels in the female rat. Stress 3:163-171.

Shors TJ, Chua C, Falduto J (2001) Sex differences and opposite effects of stress on dendritic spine density in the male versus female hippocampus. J Neurosci 21:6292-6297.

Tolin DF, Foa EB (2006) Sex differences in trauma and posttraumatic stress disorder: a quantitative review of 25 years of research. Psychol Bull 132:959-992.

Toufexis DJ, Myers KM, Bowser ME, Davis M (2007) Estrogen disrupts the inhibition of fear in female rats, possibly through the antagonistic effects of estrogen receptor $\alpha(\mathrm{ER} \alpha)$ and $\mathrm{ER} \beta$. J Neurosci 27:9729-9735.

Vazdarjanova A, McGaugh JL (1999) Basolateral amygdala is involved in modulating consolidation of memory for classical fear conditioning. J Neurosci 19:6615-6622.

Vyas A, Jadhav S, Chattarji AS (2006) Prolonged behavioral stress enhances synaptic connectivity in the basolateral amygdala. Neuroscience 143:387-393.

Wood GE, Shors TJ (1998) Stress facilitates classical conditioning in males, but impairs classical conditioning in females through activational effects of ovarian hormones. Proc Natl Acad Sci USA 95:4066-4071.

Wood GE, Beylin AV, Shors TJ (2001) The contribution of adrenal and reproductive hormones to the opposing effect of stress on trace conditioning in males versus females. Behav Neurosci 115:175-187.

Woolley CS, Gould E, Frankfurt M, McEwen BS (1990) Naturally occurring fluctuation in dendritic spine density on adult hippocampal pyramidal neurons. J Neurosci 10:4035-4039.

Yankova M, Hart SA, Woolley CS (2001) Estrogen increases synaptic connectivity between single presynaptic inputs and multiple postsynaptic CA1 pyramidal cells: a serial electron microscope study. Proc Natl Acad Sci USA 98:3525-3530.

Zurkovsky L, Brown SL, Boyd SE, Fell JA, Korol DL (2007) Estrogen modulates learning in female rats by acting directly at distinct memory systems. Neuroscience 144:26-37. 